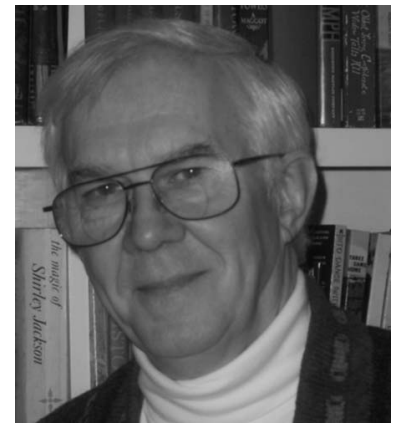

\section{Organizing Your Information}

In 2007 I wrote about a program that manages the storage and indexing of research papers on your computer (Editorial, August 2007). The program, Papers, (http:// www.mekentosj.com/papers/) written for the Apple Macintosh by two Dutch programmers, reflects their experience at working with the technical literature. The application provides a consistent method for dealing with the mass of the papers we acquire. At that time, the emphasis was on the life sciences and the only database search built into the program was PubMed. Since then they have widened the list of repositories to include Google Books, Google Scholar, IEEE Xplore, MathSciNet, and Web of Science among others. Currently Inspec, the science and engineering database, has not been included.

As I noted then, the papers are imported into the Papers library, where they can be sorted into folders, called Collections, which are based on topics defined by the user. The Collections are populated by dragging papers onto the folder. With some effort, papers existing on a computer can be identified, assembled, and organized. Then the contents of the papers, not just the title, abstract, and authors, can be searched because Papers uses the Mac's indexing system. Although a paper can be printed out at any time, most researchers will probably find the full screen mode sufficient for their purposes.

For those who are not Mac users, SPIE has provided useful resources through the SPIE Digital Library. The next time the table of contents alert for this journal appears in your inbox, open the abstract for one of the papers. (You are getting the alert and opening it, aren't you? If not, go to spiedl.org and click on the E-mail Alerts tab at the top of the page to set up your alerts.) When you open the link to an abstract of a paper (left side of the last line of the citation) a Web page for the paper will open, showing the abstract and references for the paper. In the right frame below the Full Text Options label, a number of items are listed. These links allow you to download citations, email the abstract, blog the article, or save it to a managed list of articles. For the busy researcher the most important item is the Research Toolkit. The Toolkit consists of three free browser add-on tools, Zotero, OpenURL Referrer, and Hyperwords. (A fourth extension, Google Notebook, that had been part of the toolkit is closed to new users.) By far the most interesting extension for information search and retrieval is Zotero.

Zotero is a free Firefox extension that aids in the collection, management, and citation of research sources. It is convenient because it works inside the browser and captures Web information not available through the databases accessed by Papers. The extension can be downloaded from the Web on Zotero's home page, http:// www.zotero.org/, along with a short video tour of its features. Once the extension is installed and Firefox is restarted, an access button labeled "zotero" appears in the lower right hand corner of your browser windows. (You can change this to a less obtrusive " $z$ " or eliminate it altogether, although the hot spot for the button remains.)

In comparison to Papers, Zotero captures Web pages instead of PDFs. You can save a snapshot of the page and when you retrieve it later, you get the entire page at the time you saved it. It also saves the URL, so that you can get an updated version of the page. Also, PDFs linked by the page may be attached, but it takes some work. So, Zotero contains some of the same organization features as Papers. When you click the button, a three-pane window appears at the bottom of your browser page. For example, suppose you find a paper in the alert that interests you and you would like to see more. If you are a subscriber, when you click the PDF button under Full Text Options, you can open it in Acrobat Reader.

Although it is possible to download the PDF to your computer and attach it to the item in your Zotero collection of pages, it will take some file organization on your part to eliminate any hassle. It is best to consider Zotero to be a bibliographic information collector. Once you have the information, you can either manage your own collection or use Papers.

You can gain some insight into using both Zotero and Papers from Olivia Judson, a research fellow in biology at Imperial College London and a columnist for the New York Times. She describes her usage of these two resources and discusses her attempts in information management at http://judson.blogs.nytimes.com/2008/12/16/ defeating-bedlam/.

These applications cannot substitute for our judgment as we try to drink from this veritable fire hose of papers and proceedings. But these programs provide a way to collect and organize information. Give Zotero a try-and for those who are Mac owners, I suggest you download the demo of Papers and see if it's worth it for you.

Finally, install that last item in the Research Toolkit, Hyperwords, in your browser. Its magic! 